\title{
GRBs as Probes of Massive Stars Near and Far
}

\author{
Johan P. U. Fynbo and Daniele Malesani \\ Dark Cosmology Centre, Niels Bohr Institute, University of Copenhagen, \\ DK-2100 Copenhagen O, Denmark \\ email: jfynbo@dark-cosmology.dk
}

\begin{abstract}
Long-duration gamma-ray bursts are the manifestations of massive stellar death. Due to the immense energy release they are detectable from most of the observable universe. In this way they allow us to study the deaths of single (or binary) massive stars possibly throughout the full timespan massive stars have existed in the Universe. GRBs provide a means to infer information about the environments and typical galaxies in which massive stars are formed. Two main obstacles remain to be crossed before the full potential of GRBs as probes of massive stars can be harvested: $i$ ) we need to build more complete and well understood samples in order not to be fooled by biases, and ii) we need to understand to which extent GRBs may be intrinsically biased in the sense that they are only formed by a limited subset of massive stars defined by most likely a restricted metallicity interval. I describe the status of an ongoing effort to build a more complete sample of long-duration GRBs with measured redshifts. Already now we can conclude that the environments of GRB progenitors are very diverse with metallicities ranging from solar to a hundredth solar and extinction ranging from none to $A_{V}>5$ mag. We have also identified a sightline with significant escape of Lyman continuum photons and another with a clear $2175 \AA$ extinction bump.
\end{abstract}

Keywords. gamma rays: bursts - galaxies: distances and redshifts

\section{Introduction}

GRBs were discovered serendipitously in the late 1960's and first reported to the astrophysical community in the early 1970's (Klebesadel et al. 1973). For a long time their nature remained a mystery (e.g., Nemiroff 1994). For a review of the first decades of GRB research where only the prompt $\gamma$-ray emission was known see Fishman \& Meegan (1995). The major breakthrough came in 1997 with the ability to determine celestial positions of GRBs thanks to the BeppoSAX satellite and the discovery of long-lived Xray, optical and radio afterglows (e.g., Costa et al. 1997; van Paradijs et al. 1997; Frail et al. 1997). For a review of the early years of the so called afterglow era (after 1997) see van Paradijs et al. (2000).

GRBs come in at least two variants defined by their duration in the $\gamma$-ray band. The short bursts have duration less than about 2 se and the long bursts longer than this (Kouveliotou et al. 1993). In the reminder of this review we will only discuss the long duration bursts. The association between long-duration GRBs (LGRBs hereafter) and massive stars and hence the link between LGRBs and on-going massive star-formation found its first empirical basis with the detection of the first host galaxies (e.g., Hogg et al. 1999). Subsequently, the evidence was further strengthened with the discovery of supernovae (SNe) associated with LGRBs (Galama et al. 1998; Hjorth et al. 2003; Stanek et al. 2003; Malesani et al. 2004; Sollerman et al. 2006; Pian et al. 2006). For a recent review of the LGRB/SN association see Woosley \& Bloom (2006). 
Because of the link between LGRBs and massive stars and due to the fact that GRBs can be detected from both the most distant and the most dust obscured regions in the universe LGRBs were quickly identified to be very promising tracers of star-formation throughout cosmic history (e.g., Wijers et al. 1998). However, this potential has so far not really resulted in an improved census of the locations of massive stars due to complications discussed in the next section.

A major issue currently under discussion is if LGRBs are unbiased tracers of star formation. More precisely, it is not clear if LGRBs are caused by the same (small) fraction of all dying massive stars (unbiased tracers), or if LGRBs only trace a limited segment defined by parameters such as, e.g., metallicity or circumstellar density (biased tracers).

The currently operating Swift satellite (Gehrels et al. 2004) has revolutionized LGRB research with its frequent, rapid, and precise localization of LGRBs. Now it is for the first time possible in practice to use LGRBs as powerful probes. It is mandatory that this potential is exploited while Swift is still operating (at least until 2010).

\section{Complications in the use of LGRBs as tracers of massive stars}

\subsection{Dark bursts and incomplete samples}

A crucial issue when using LGRBs (or any other class of tracer) is sample selection. Whereas the detection of the LGRB itself poses no bias against dust obscured massive stars this is not the case for the softer afterglow emission which is crucial for obtaining the precise localization as well as measuring redshifts (see, e.g., Fiore et al. 2007).

In the samples of LGRBs detected with satellites prior to the currently operating Swift satellite the fraction of LGRBs with detected optical afterglows was only about 30\% (Fynbo et al. 2001; Lazzati et al. 2002). Much of this incompleteness was caused by random factors such as weather or unfortunate celestial positions of the bursts, but some remained undetected despite both early and deep limits. It is possible that some of these so called "dark bursts" could be caused by LGRBs in very dusty environments (Groot et al. 1998) and hence the sample of LGRBs with detected optical afterglows could very well be systematically biased against dust obscured star formation (see also Jakobsson et al. 2004a; Rol et al. 2005; Rol et al. 2007 for recent discussions of the dark bursts).

In any case, such a high incompleteness imposes a large uncertainty on statistical studies based on LGRB host galaxies derived from these early missions. It should be stressed that the conclusions based on these samples may only be relevant for a minority of all LGRBs. Due to the much more precise and rapid localization capability of Swift it is now possible to build much more complete samples.

\subsection{Are some LGRBs not associated with massive stellar death?}

Recently, it has been found that some LGRBs are not associated with SNe, namely GRB 060505 (Fynbo et al. 2006a; Ofek et al. 2007) and GRB 060614 (Fynbo et al. 2006a; Della Valle et al. 2006; Gal-Yam et al. 2006). This means that either some massive stars die without producing SNe brighter than about $M_{\mathrm{V}}=-13.5$ (Fynbo et al. 2006a, Della Valle et al. 2006) or, alternatively, some LGRBs are caused by other mechanisms than collapsing massive stars (Gal-Yam et al. 2006; Ofek et al. 2007).

At least for the case of GRB 060505 the evidence points to the former. The burst was located in a star-forming region in a relatively low metallicity region in the outer part of a spiral host (Fynbo et al. 2006a; Ofek et al. 2007; Thöne et al. 2008; see also Fig. 1). The burst itself displayed a significant spectral lag, which has so far never been seen for the short bursts that are believed to originate from merging compact objects (McBreen et al. 2008; Norris \& Bonnell 2006). If some LGRBs indeed are caused by other progenitors 
than massive stars then a new classification that can distinguish between LGRBs from massive stars and those from other mechanisms is required. So far no such scheme has been found (see Gehrels et al. 2006).

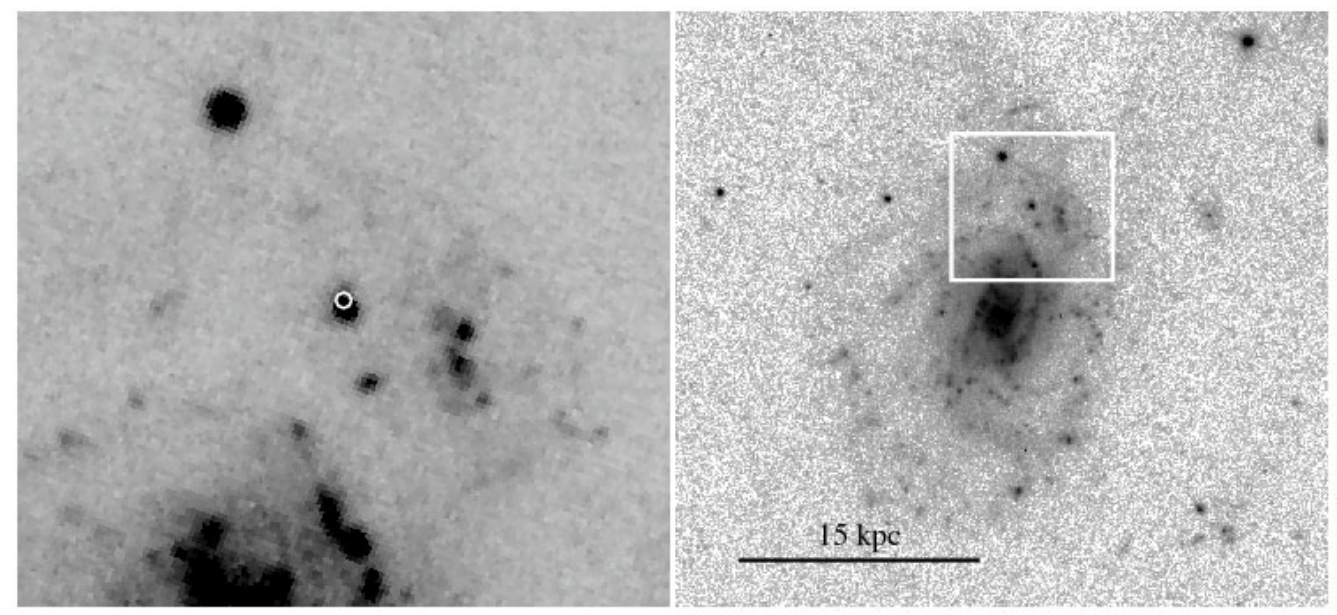

Figure 1. The host galaxy of GRB 060505 as observed with the HST (Ofek et al. 2007; Thöne et al. 2008). The white circle shows the error-circle of the burst consistent with a star-forming region in a spiral arm. This is strong evidence that the progenitor was a massive star. The properties of this host is also within the range found for other LGRBs. As an example, the host galaxy of GRB 050824 is very similar in terms of luminosity, $R_{23}$ and star-formation rate (Sollerman et al. 2007), and the location within the host is similar to the location of other LGRBs in spiral hosts (Fynbo et al. 2000a; Sollerman et al. 2002; Le Floc'h et al. 2002; Jakobsson et al. 2005a).

\subsection{The contamination from chance projections}

The first important question to ask is: are LGRB host galaxies operationally well-defined as a class? In terms of an operational definition the case is not so clear. If we define the host galaxy of a particular burst to be the galaxy nearest to the line-of-sight, we need to worry about chance projection (Band \& Hartmann 1998). In the majority of cases where an optical afterglow has been detected and localized with sub-arcsecond accuracy and where the field has been observed to deep limits a galaxy has been detected within an impact parameter less than 1 arcsec (see e.g., Bloom et al. 2002 and Fig. 2 for an example). The probability for this to happen by chance depends on the magnitude of the galaxy. The number of galaxies per $\operatorname{arcmin}^{2}$ has been well determined to deep limits in the Hubble deep fields. In Fynbo et al. (2000b, their Fig. 2) the galaxy counts in the R and I bands based on the HDF-South can be found. To limits of $\mathrm{R}=24,26$ and 28 there are about 2, 6 and 13 galaxies $\operatorname{arcmin}^{-2}$. Hence, the probability to find a $\mathrm{R}=24$ galaxy by chance in an error circle with radius 0.5 arcsec is about $4 \times 10^{-4}$. For a $\mathrm{R}=28$ galaxy the probability is about $3 \times 10^{-3}$. If the error circle is defined only by the $\mathrm{X}$-ray afterglow with a radius of 2 arcsec in the best cases then we expect a random $R=24$ and $R=28$ galaxy in $0.6 \%$ and $5 \%$ of the error circles. For a sample of a few hundred LGRBs chance projection should hence not be a serious concern for LGRBs localized to sub-arcsecond precision, but for error-circles with radius of a few arcseconds we expect a few chance projections. In some cases it may be possible to eliminate the chance projects, e.g., based on conflicting redshift information from the afterglow and proposed host, but in general not. 


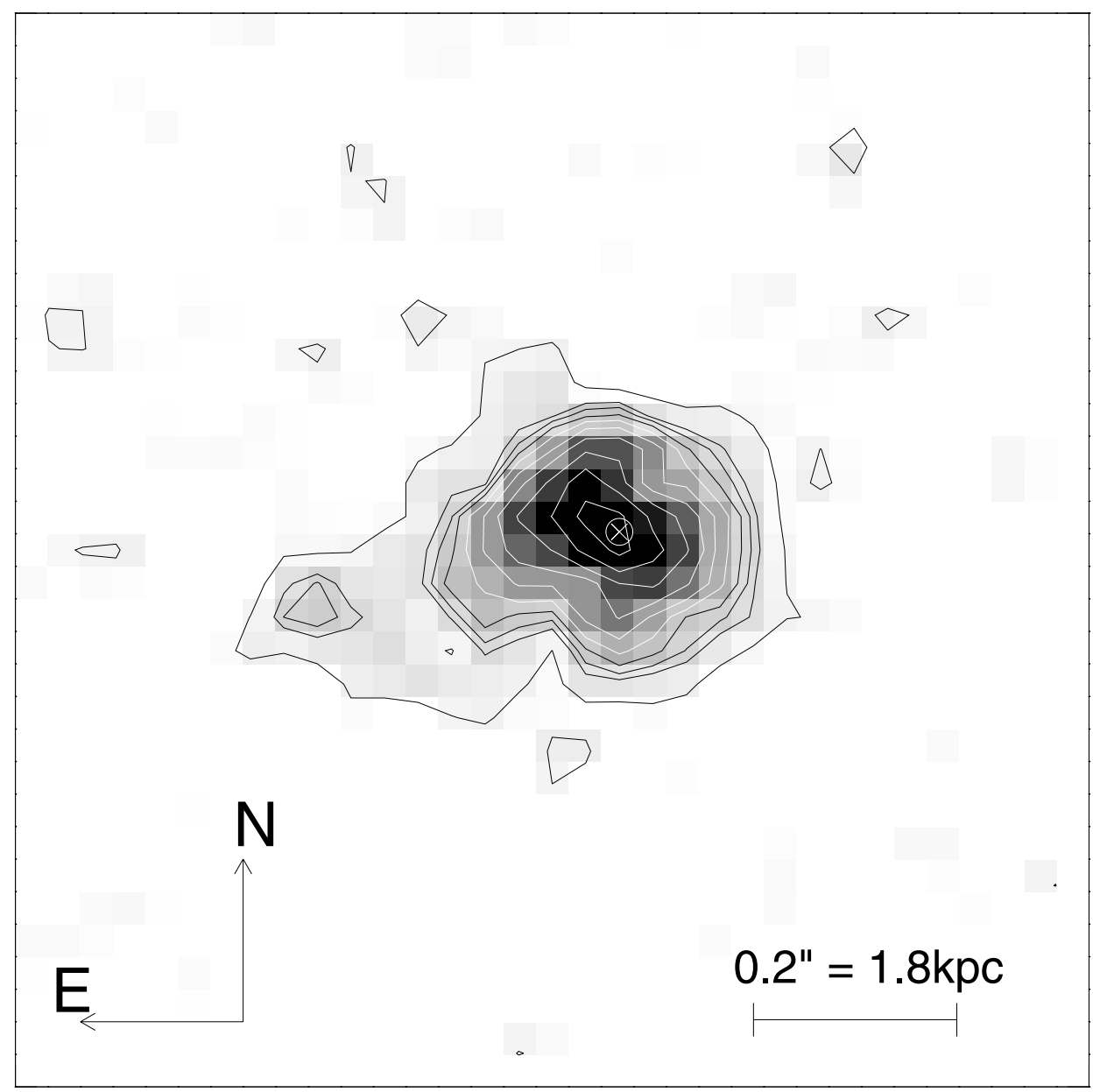

Figure 2. The $1 \times 1 \operatorname{arcsec}^{2}$ field around the host galaxy of the $z=2.33$ HETE-2 GRB 021004 observed with the HST (from Fynbo et al. 2005). The LGRB went off near the center of the galaxy. The position of the LGRB is marked with a cross and an error circle and it coincides with the centroid of the galaxy to within a few hundredths of an arcsec. In cases like this there is no problem in identifying the correct host galaxy. However, in cases of bursts localized to only a few arcsec accuracy chance projection needs to be considered.

\section{Conclusions based on pre-Swift samples}

Despite the complications mentioned above, the previous decade of LGRB host galaxy studies has after all taught us a lot about LGRBs and their link to massive star formation (see van Paradijs et al. 2000 and Djorgovski et al. 2003 for early reviews). LGRB hosts were early on found to be predominantly faint, blue star-forming galaxies (Hogg et al. 1999). The early studies found that these properties of LGRB hosts were consistent with the expectation if LGRBs are unbiased tracers of star-formation (Mao et al. 1998; Hogg et al. 1999). It was also realized early on that LGRBs offer a unique possibility to locate and study star-formation activity in dwarf galaxies at $z>2$ (Jensen et al. 2001). This is basically impossible with any other currently existing method. The star-formation rates were found to be modest, but the specific star-formation rates among the highest ever found (Christensen et al. 2004). LGRB hosts are hence often in a starburst state. 
Later evidence indicated that LGRBs maybe related only to massive stars with metallicity below a certain threshold. The first evidence for this came with the realization the LGRB hosts were fainter and bluer than expected according to certain models about the nature of the galaxies dominating the integrated star-formation activity (Le Floc'h et al. 2003, 2006; Tanvir et al. 2004). Nevertheless, it has recently been pointed out by Priddey et al. (2006) that "there is sufficient uncertainty in models and underlying assumptions, as yet poorly constrained by observation (e.g., the adopted dust temperature) that a correlation between massive, dust-enshrouded star formation and GRB production cannot be firmly ruled out." (see also Michałowski et al. 2008 concerning the issue of dust temperature). Further circumstantial evidence for a preference towards low metallicity came from the observation that Lyman- $\alpha$ emission seemed to be ubiquitous for LGRBs hosts (Fynbo et al. 2003; Jakobsson et al. 2005b).

Lately, evidence from hosts of more local LGRBs seems to point in the same direction. Several studies have found that local LGRBs hosts tend to be faint and metal poor although a caveat for some of these studies is the difficulty of using the strong line metallicity indicators like $R_{23}$ to derive robust metallicities (Prochaska et al. 2004; Sollerman et al. 2005; Gorosabel et al. 2005; Stanek et al. 2006; Wiersema et al. 2007). However, nearly all of these studies targeted very incomplete pre-Swift samples and this raises the question whether the predominance of faint, metal poor hosts can be explained by, e.g., a bias against metal rich hosts and hence more dust obscured LGRB afterglows.

A very important result is that LGRBs and core-collapse SNe are found in different environments (Fruchter et al. 2006). The same study also found that LGRB host galaxies at $z<1$ are fainter than the host galaxies of core-collapse SNe. This study is also based on incomplete pre-Swift samples, but as the SNe samples are if anything more biased against dusty regions than LGRBs this result does seem to be substantial evidence that LRBs are biased towards massive stars with relatively low metallicity. Wolf \& Podsiadlowski (2007) however, find, based on an analysis of the Fruchter et al. (2006) data, that the metallicity threshold cannot be below half the solar metallicity. Concerning the different environments of core-collapse SNe and LGRBs it has recently been found that type Ic SNe have similar positions relative to their host galaxy light profiles as LGRBs, whereas all other SN types have a similar distribution, less centred on their host light than LGRBs and SN Ic's (Kelly et al. 2007). Larsson et al. (2007) find that the different distributions of different SN types relative to their host light can be naturally explained by assuming different mass ranges for the typical progenitor stars: $\gtrsim 8 \mathrm{M}_{\odot}$ for typical core-collapse $\mathrm{SNe}$ and $\gtrsim 20 \mathrm{M}_{\odot}$ for LGRB progenitors. The picture is complicated by the finding that type Ic SNe typically are found in substantially more metal rich environments than LGRBs (Modjaz et al. 2008, and in these proceedings). It is well established that WRstars become more abundant with increasing metallicity - opposite to LGRBs that if anything are biased towards low metallicity. Taken together these findings suggest that progenitors of LGRBs and "normal" type Ic SNe are two different subsets of the $\gtrsim 20 \mathrm{M}_{\odot}$ stars. For a thorough discussion of the relation between WR stars, SN Ic's and LGRBs we refer to Crowther (2007).

\section{Building a complete sample of Swift LGRBs}

The following is to a large extent based on Fynbo et al. (2007). The Swift satellite has been operating for about three years and is far superior to previous GRB missions. The reason for this is the combination of several factors: $i$ ) it detects LGRBs at a rate of about two bursts per week about an order of magnitude larger than the previous successful BeppoSAX and HETE-2 missions; ii) with its X-Ray Telescope (XRT) it localizes the 
bursts with a precision of about 5 arcsec also orders of magnitude better than previous missions; iii) it has a much shorter reaction time, allowing the study of the evolution of the afterglows literally seconds after the burst, sometimes during the prompt $\gamma$-ray emission itself. The Swift mission is funded at least until 2010. A crucial objective is to secure a large sample, as complete as possible, of LGRB afterglows while Swift is still operating. More concretely, rather than including all Swift detected GRBs, it is more optimal to concentrate on those LGRB afterglows with favourable observing conditions. Our group uses the following sample criteria:

(a) XRT afterglow detected within $12 \mathrm{hr}$

(b) Small foreground Galactic extinction: $A_{V}<0.5 \mathrm{mag}$

(c) Favourable declination: $-70<$ Dec $<70$

(d) Sun distance larger than $55^{\circ}$

By introducing these constraints, we are not biasing the sample towards optically bright afterglows, but we select a sample for which useful follow-up observations are likely to be secured.

About 50\% of all Swift LGRBs do not fulfill these criteria, primarily because Swift, for technical reasons, has to point close to the Sun a significant fraction of the time. For bursts fulfilling the above criteria, we make every possible effort to detect optical and near-infrared afterglows and to measure their redshifts. As shown below, we have been very successful in this effort, using mainly the ESO VLT. Redshifts, or more generally spectroscopic observations, are crucial for almost all LGRB-related science. The most important science cases for which spectroscopy is critical are listed below:

- Determining the luminosity function for LGRBs (prompt emission as well as afterglows).

- Determining the redshift distribution of LGRBs and using LGRBs as tracers for the cosmic star-formation history (Jakobsson et al. 2006a; Fiore et al. 2007).

- Studying the host galaxies, in particular those faint, high-redshift galaxies that are unlikely to be found and studied with other methods (e.g., Vreeswijk et al. 2004).

- Studying LGRB-selected absorption-line systems (e.g., Jakobsson et al. 2004b; Prochter et al. 2006).

- Characterizing the dust extinction curves of high- $z$ galaxies (e.g., Jensen et al. 2001; see also Fig. 4).

- Determining the Lyman continuum escape fraction from high- $z$ galaxies (Chen et al. 2007, see also Fig. 5).

- Spotting very high redshift LGRBs (e.g., Kawai et al. 2006; Ruiz-Velasco et al. 2007).

- Probing cosmic chemical evolution with LGRBs (e.g., Savaglio 2006; Fynbo et al. 2006b; Prochaska et al. 2007).

- Studying if LGRBs can be used for cosmography (e.g., Ghirlanda et al. 2004).

Since the launch of Swift, we have had programmes running at the VLT with the aim of securing redshifts for Swift LGRBs. The status at the time of writing is that 109 Swift LGRBs fulfilled our selection criteria. For 57 of these a redshift measurement has been secured (see Fig. 6). The VLT has been the dominant single contributor in all LGRB redshift measurements, providing around $40 \%$ of the secure redshifts to date. The redshift of the most distant known GRB 050904 at $z=6.295$ was measured with the Subaru telescope (Kawai et al. 2006). Most of the other redshifts have been measured using other 6-10 m telescopes (Keck, Gemini, Subaru, Magellan). This is contrary to the expectations prior to the launch of Swift, where it was suspected that Swift itself, or at least 2-4-m telescopes, would be able to measure most of the redshifts. However, optical afterglows turned out to be much fainter at early times than anticipated. As we show in Fig. 3, the majority of the afterglows are fainter than $R=20$ when a slit can be placed 


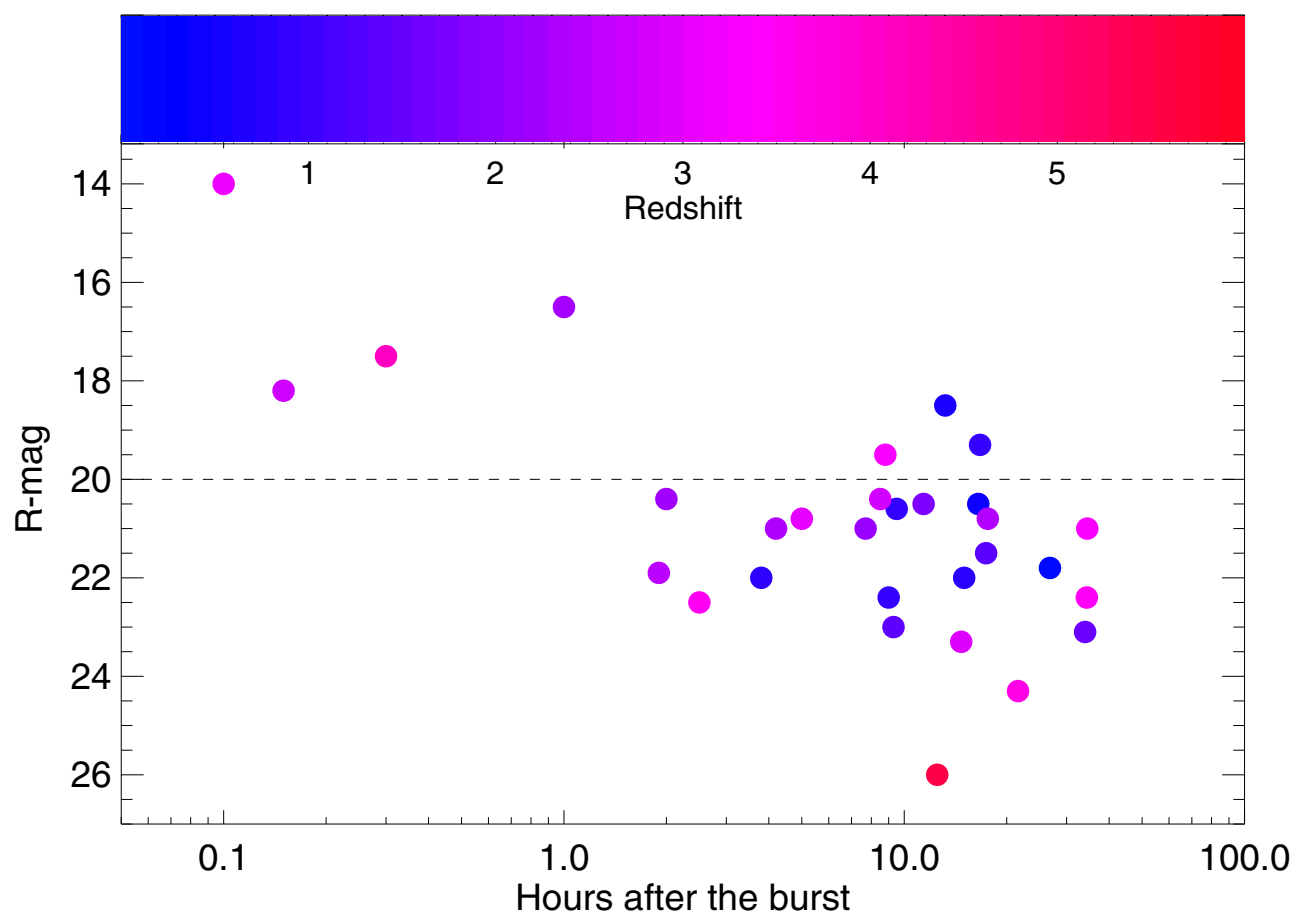

Figure 3. The R-band magnitude of the optical afterglows as a function of the time after the burst at which the spectroscopic observations were obtained. Only included are Swift bursts for which we have measured the redshift (using primarily the VLT, but also NOT, WHT and Gemini). The bar at the top indicates the code for the measured redshifts. The dashed line marks a magnitude of $R=20$ which is roughly the spectroscopic limit for 2-4-m telescopes for detecting absorption lines. As seen, most afterglows are fainter than this limit when observable.

on them. $R=20$ is, in our experience, the limit for spectroscopic redshift determination using 2-4-m telescopes (typically, no more than 1-2 hr exposure time is available for observing LGRB afterglows). Several optical afterglows are already fainter than $R=22$ a few hours after the bursts. Hence, 6-10 m telescopes are crucial for securing redshifts for the majority of Swift LGRBs.

The fact that in particular the VLT, but also other 6-10 m telescopes, have made tremendous efforts to secure redshifts means that we now have a much higher redshift completion than for pre-Swift samples. But it is clear that we will not get redshifts for all bursts from spectroscopy of the afterglows for multiple reasons. In about 20-30\% of the triggers we are not able to measure the redshift either due to lack of lines (probably bursts at redshifts between 1 and 2, see Fig. 6), bad weather or because the afterglow has faded too much before it is observable from Paranal. For these bursts our only chance of measuring the redshift is via spectroscopy of the host galaxy. We have also pursued this route extensively in an ESO large program (PI Hjorth). This is a challenging task due to the faintness of these systems, and the analysis of these data is still ongoing, but we have already determined a number of redshifts (included in Fig. 6).

\subsection{The redshift distribution of Swift LGRBs: current status}

The first conclusion from Fig. 6 is that Swift LGRBs are very distant. Swift LGRBs are more distant than LGRBs from previous missions due to the higher sensitivity of the satellite to the lower energies prevalent in the more distant events (Fiore et al. 2007). 


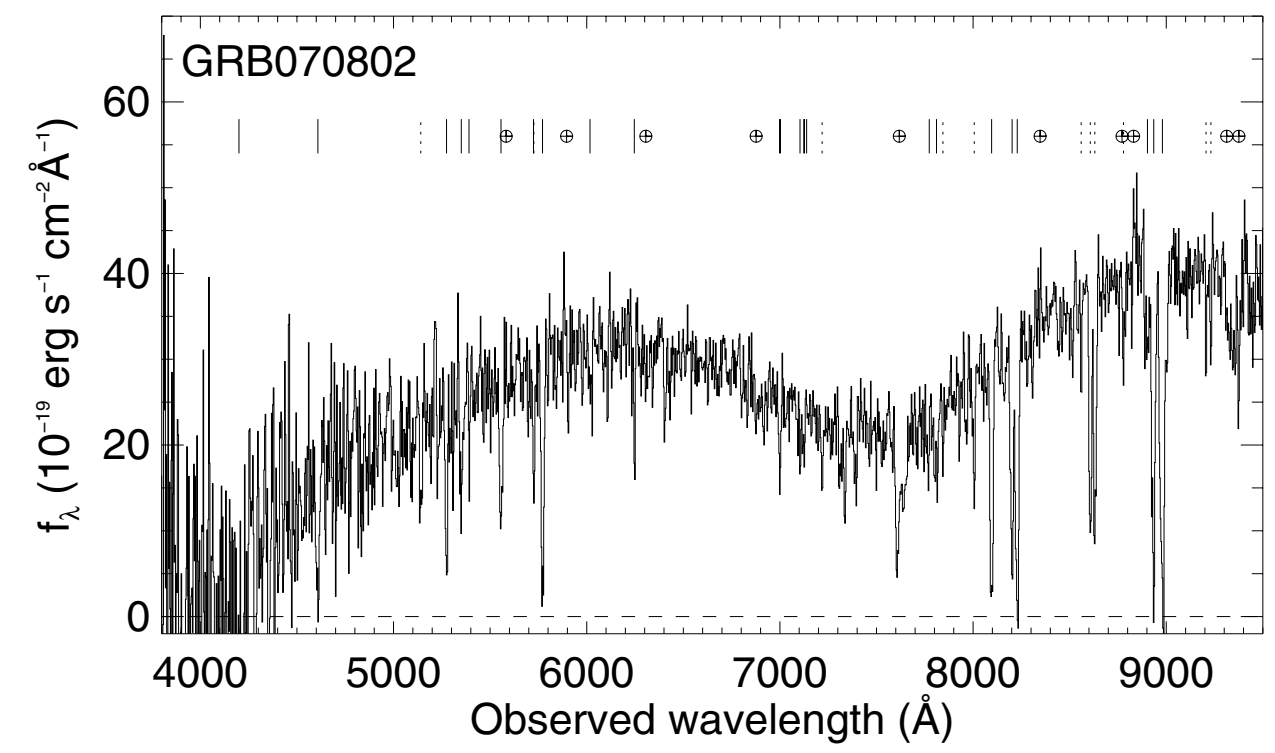

Figure 4. The VLT/FORS2 spectrum of the afterglow of GRB 070802 (Elíasdóttir et al., in preparation). Plotted is the flux-calibrated spectrum against observed wavelength. Metal lines at the host redshift are marked with full-drawn lines whereas the lines from two intervening systems are marked with dotted lines. The broad depression centred around $7500 \AA$ is caused by the $2175 \AA$ extinction bump in the host system at $z_{\mathrm{abs}}=2.4549$.

The median and mean redshift are now both 2.3, while for previous missions it was closer to 1 (Jakobsson et al. 2006a). The record holder is $z=6.295$ (Kawai et al. 2006). It is striking how events at redshifts as large as 6 can be detected within such a small sample. For comparison, only a few QSOs are detected at similar distances out of a sample of hundred thousand QSOs. Remarkably, the redshift distribution, measured for just over $50 \%$ of all bursts, is consistent with the redshift distribution predicted if LGRBs are unbiased tracers of star formation (see, e.g., Jakobsson et al. 2006a and http://www.darkcosmology.dk/ pallja/GRBsample.html for a regularly updated analysis).

\subsection{HI column densities}

The HI column density distribution for LGRB sightlines is extremely broad. It covers a range of about 5 orders of magnitude from $\sim 10^{17} \mathrm{~cm}^{-2}$ (Fig. 5, Chen et al. 2007) to nearly $10^{23} \mathrm{~cm}^{-2}$ (Jakobsson et al. 2006b). I still remain to be understood if this distribution is representative of the intrinsic distribution of HI column densities towards massive stars in galaxies or if the distribution is rather controlled by the ionizing emission from the afterglows themselves. In any case, as pointed out by Chen et al. (2007) the HI column density distribution provides an upper limit to the escape fraction of Lyman continuum emission from star-forming galaxies.

\subsection{Metallicities}

Afterglow spectroscopy often allows us to measure the metallicity of the line-of-sight in the host galaxy. In Fig. 7 we plot the metallicities along LGRB sightlines together with metallicities derived from QSO damped Lyman- $\alpha$ absorbers (QSO-DLAs). Here it can be seen that LGRBs are more metal rich than QSO-DLAs at similar redshifts. Some of the LGRB sightlines are almost as metal rich as the Lyman-break galaxies at similar redshifts (Pettini et al. 2001). The shift in metallicity relative to QSO-DLAs 


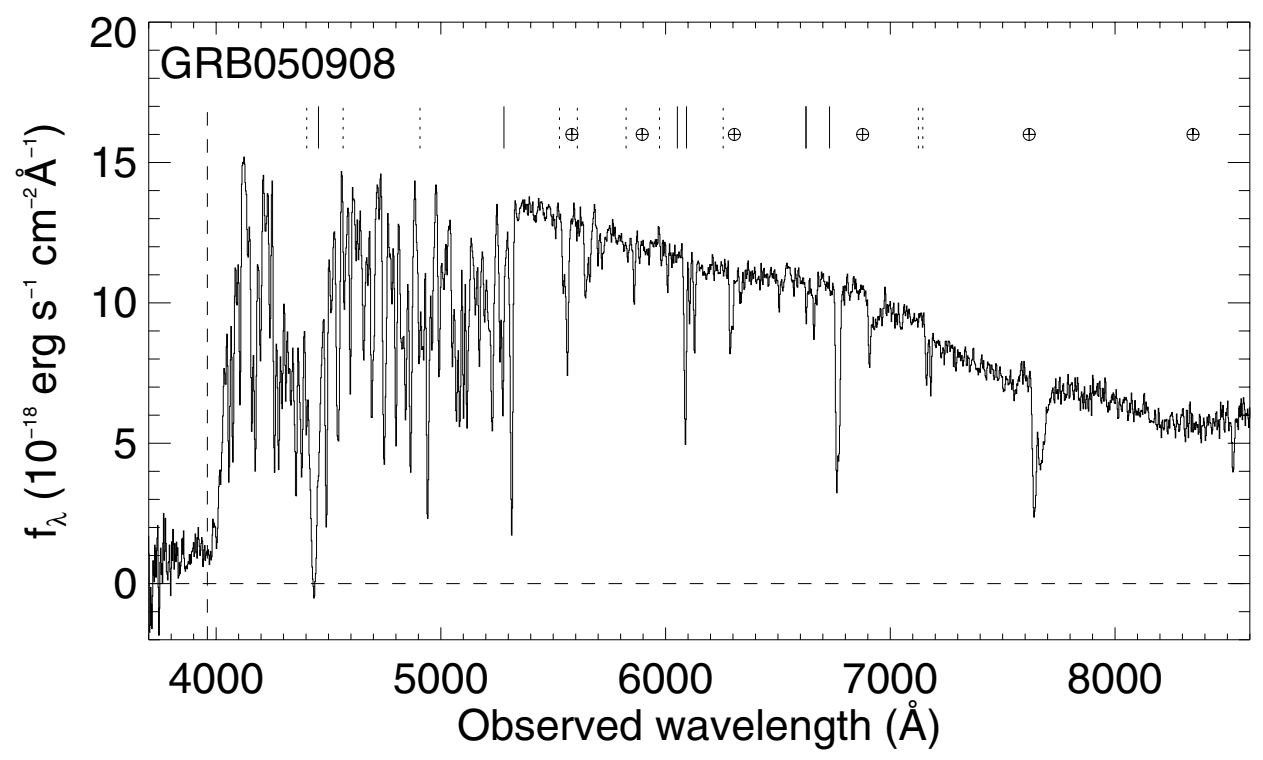

Figure 5. The VLT/FORS2 spectrum of the afterglow of GRB 050908 (Smette et al., in preparation). Plotted is the flux-calibrated 1-dimensional spectrum against observed wavelength. The vertical dashed line shows the position of the Lyman limit at the redshift of the GRB $(z=3.343)$. As seen, there is clear excess flux below the Lyman limit.

can be understood from the different selection functions of the (star-formation selected) LGRB-DLAs and the (HI cross-section selected) QSO-DLAs combined most likely with metallicity gradients in high- $z$ galaxies (Fynbo et al. 2008). Hence, most likely LGRBs will give a reasonably unbiased census of where the massive stars are located, at least at $z>2$ (Fynbo et al. 2006b).

\subsection{Extinction}

In addition to HI column densities, metal and molecular abundances and kinematics, the afterglow spectra also provide information of the extinction curves. The intrinsic spectrum of the afterglow is from theory predicted to be a power-law and therefore any curvature or other broad features in the spectrum can be interpreted as being due to features in the extinction curve. So far, almost all the extinction curves derived for LGRB host galaxy sightlines have been consistent with an extinction curve similar to that of the SMC. Recently, we obtained the clearest yet detection of the $2175 \AA$ bump known from the Milky Way in a $z=2.45$ LGRB (Eliasdottir et al., in preparation and Fig. 4). This LGRB absorber also has unusually strong metal lines suggesting that the presence of the $2175 \AA$ extinction bump is related to a high metallicity. However, we have examples of LGRBs with nearly solar metallicity for which the bump is not seen so it seems that metallicity is not the only parameter controlling the presence of the $2175 \AA$ extinction bump. Concerning the amount of extinction the LGRB sightlines vary from no extinction (e.g., GRB050908, Fig. 5) to $A_{V}>5$ mag (e.g., GRB070306, Jaunsen et al. 2008).

\section{Conclusions and outlook}

Spectroscopy of LGRB afterglows provides redshifts and information on the ISM properties for the population of galaxies containing the bulk of the high-redshift massive stars. 


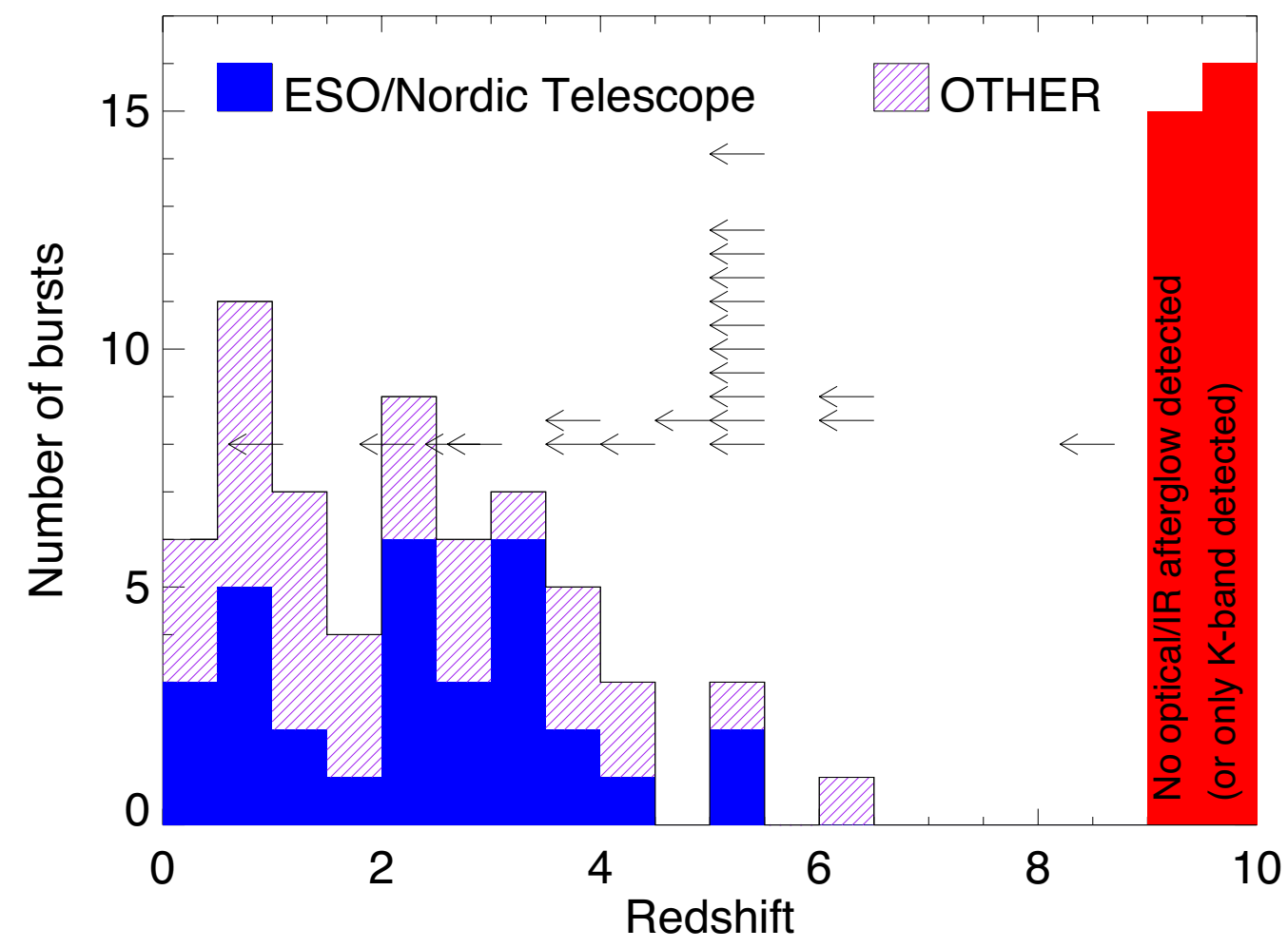

Figure 6. Redshift distribution (up to October 2007) of 109 Swift LGRBs localized with the X-ray telescope and with low foreground extinction $A_{V}<0.5$. Of the 58 measured redshifts, our group has measured nearly half (25, shown in black). As shown, the VLT is the dominant source of redshifts in the Swift era (four of the black bursts in the histogram are also from the Nordic Optical Telescope). Bursts, for which only an upper limit on the redshift could be established so far, are indicated by arrows. Note that it is also difficult to secure redshifts for GRBs in the desert between $z=1$ and $z=2$. The grey histogram at the right indicates the 27 bursts for which no optical/J/H afterglow was detected and hence no redshift constraint could be inferred (see Ruiz-Velasco et al. 2007 for a full discussion).

We are currently working on securing this information for a complete sample of Swift LGRBs. Already now we can conclude that the environments of LGRB progenitors are very diverse with metallicities ranging from solar to a hundredth solar and extinction ranging from none to $A_{V}>5$ mag. We have also identified a sightline with significant escape of Lyman continuum photons and another with a clear $2175 \AA$ extinction bump.

Even though the completeness of the current Swift sample in terms of detections of optical afterglows $(\sim 75 \%)$, redshift determinations $(\sim 50 \%)$ and host galaxy detections $(\sim 80 \%)$ is much higher than for LGRBs from previous missions we still need to do better - preferably all three fractions should be $\gtrsim 80 \%$. We also need to improve the understanding of the link between LGRBs and massive stars. The SN-less GRBs GRB 060505 and GRB 060614 show that either some LGRBs are unrelated to massive stellar death or some massive stars die without causing SNe. The currently unfolding SN2008D/XRF080109 shows that there may be much more frequent bursts with softer prompt emission bridging the gap (in terms of both burst, SN and host properties) between GRBs and normal Ic SNe (e.g., Soderberg et al. 2008; Malesani et al., 2008). 


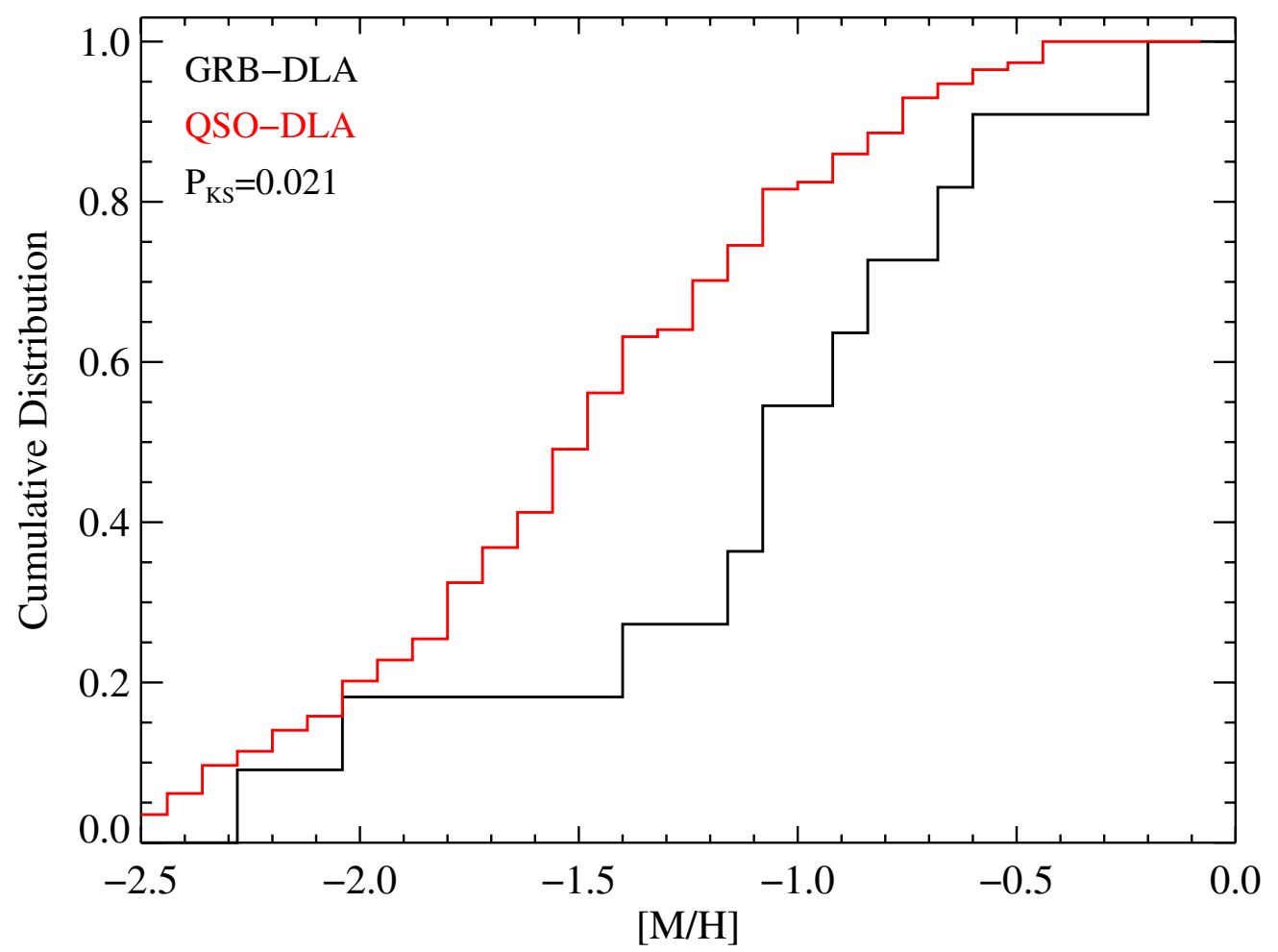

Figure 7. The histograms show the cumulative distribution of QSO-DLA and LGRB-DLA metallicities in the statistical samples compiled by Prochaska et al. (2003) and Prochaska et al. (2007). As seen, the LGRB-DLA metallicities are systematically higher than the QSO-DLA metallicities. See also Fynbo et al. (2008) for a simple model of these two distributions.

\section{Acknowledgements}

We thank our collaborators and the Swift team for carrying out such a wonderful experiment. The Dark Cosmology Centre is funded by the DNRF.

\section{References}

Band, D. L. \& Hartmann, D. H. 1998 ApJ, 493, 555

Bloom, J. S., Kulkarni, S. R., \& Djorgovski, S. G. 2002 AJ, 123, 1111

Chen, H.-W., Prochaska, J. X., \& Gnedin, N. Y. 2007 ApJ, 667, L125

Christensen, L., Hjorth, J., \& Gorosabel, J. 2004 A\&SA, 425, 913

Costa, E., Frontera, F., Heise, J., et al. 1997 Nat, 387, 783

Crowther, P. A. 2007 ARA $\& A, 45,177$

Della Valle, M., Chincarini, G., Panagia, N., et al. 2006 Nat, 444, 1050

Djorgovski, S. G., Kulkarni, S. R., Frail, D. A., et al. 2003, in: P. Guhathakurta (ed.), Discoveries and Research Prospects from 6-10m Class Telescopes (Bellingham: SPIE) Proc. SPIE, Vol. 4834, 238

Fiore, F., Guette, D., Piranomonte, S., et al. 2007 A\&A, 470, 515

Fishman, G. J. \& Meegan, C. A. 1995 ARAESA, 33, 415

Frail, D. A., Kulkarni, S. R., Nicastro, L., et al. 1997 Nat, 389, 261

Fruchter, A. S., Levan, A., Strolger, L., et al. 2006 Nat, 441, 463

Fynbo, J. P. U., Holland, S. T., Andersen, M. I., et al. 2000a ApJ, 542, L89

Fynbo, J. P. U., Freudling, W., \& Møller, P. 2000b A\&A, 355, 37 
Fynbo, J. P. U., Jensen, B. L., Gorosabel, J., et al. 2001 A $\& A, 369,373$

Fynbo, J. P. U., Jakobsson, P., Møller, P., et al. 2003 A\& A, 406, L63

Fynbo, J. P. U., Gorosabel, J., Smette, A., et al. 2005 ApJ, 633, 317

Fynbo, J. P. U., Starling, R. L. C., Ledoux, C., et al. 2006 A\&A, 451, L47

Fynbo, J. P. U., Watson, D., Thöne, C. C., et al. 2006 Nat, 444, 1047

Fynbo, J. P. U., Vreeswijk, P., Jakobsson, P., et al. 2007 ESO-Messenger, 130, 43

Fynbo, J. P. U., Prochaska, J. X., Sommer-Larsen, J., et al. 2008 ApJ, submitted (arXiv:0801.3273)

Galama, T. J., Vreeswijk, P. M., van Paradijs, J., et al. 1998 Nat, 395, 670

Gal-Yam, A., Fox, D. B., Price, P. A., et al. 2006 Nat, 444, 1053

Gehrels, N., Chincarini, G., Giommi, P., et al. 2004 ApJ, 611, 1005

Gehrels, N., Norris, J. P., Barthelmy, S. D., et al. 2006 Nat, 444, 1044

Ghirlanda, G., Ghisellini, G., Lazzati, D., \& Firmani, C. 2004 ApJ, 613, L13

Gorosabel, J., Pérez-Ramírez, D., Sollerman, J., et al. 2006 A\&SA, 444, 711

Groot, P., Galama, T. J., van Paradijs, J., et al. 1998 ApJ, 493, L27

Hjorth, J., Sollerman, J., Møller, P., et al. 2003 Nat, 423, 847

Hogg, D. W. \& Fruchter, A. S. 1999 ApJ, 520, 54

Jakobsson, P., Hjorth, J., Fynbo, J. P. U., et al. 2004a ApJ, 617, L21

Jakobsson, P., Hjorth, J., Fynbo, J. P. U., et al. 2004b A\& A, 427, 785

Jakobsson, P., Frail, D. A., Fox, D. B., et al. 2005a ApJ, 629, 45

Jakobsson, P., Björnsson, G., Fynbo, J. P. U., et al. 2005b MNRAS, 362, 245

Jakobsson, Levan, A., Fynbo, J. P. U., et al. 2006a A\&SA, 447, 897

Jakobsson, Fynbo, J. P. U., Ledoux, C., et al. 2006b A\&A, 460, L13

Jaunsen, A. O., Rol, E., Watson, D. J., et al. 2008 ApJ, in press (arXiv:0803.4017)

Jensen, B. L., Fynbo, J. P. U., Gorosabel, J., et al. 2001 A\&A, 370, 909

Kawai, N., Kosugi, G., Aoki, K., et al. 2006 Nat, 440, 184

Kelly, P. L., Kirshner, R. P., \& Pahre, M. 2008, submitted (arXiv:0712.0430)

Klebesadel, R. W., Strong, I. B., \& Olson, R. A. 1973, ApJ, 182, L85

Kouveliotou, C., Meegan. C. A., Fishman, G. J., et al. 1993, ApJ, 413, L101

Larsson, J., Levan, A. J., Davies, M. D., \& Fruchter, A. S. 2007 MNRAS, 376, 1285

Lazzati, D., Covino, S., \& Chisellini, G. $2002 \operatorname{MNRAS,330,583}$

Le Floc'h, E., Duc, P.-A., Mirabel, I. F., et al. 2002 ApJ, 581, L81

Le Floc'h, E., Duc, P.-A., Mirabel, I. F., et al. 2003 A\& $A$, 400, 499

Le Floc'h, E., Charmandaris, V., Forrest, W. J., et al. 2006 ApJ, 642, L636

Mao, S. \& Mo, H. J. 1998 ApJ, 339, L1

Malesani, D., Tagliaferri, G., Chincarrini, G., et al. 2004 A\&A, 609, L5

Malaseni, D., Fynbo, J. P. U., Hjorth, J., et al. 2008, ApJL, submitted (arXiv:0805.1188)

McBreen, S., Foley S., Watson D., et al. 2008 ApJL, ApJ, 667, L85

Michałowski, M., Hjorth, J., Castro-Cerón, \& J. M., Watson, D. 2008 ApJ, 672, 817

Modjaz, M., Kewley, L., Kirshner, R. P., et al. 2008 AJ, 135, 1136

Nemiroff, R. J. 1994, Comments on Astrophysics, 17, 189 (astro-ph/9402012)

Norris, J. P. \& Bonnell, J. T. 2006 ApJ, 643, 266

Ofek, E. O., Cenko, S. B., Gal-Yam, A., et al. 2006 ApJ, 662, 1129

Pettini, M., Shapley, A. E., Steidel, C. C., et al. 2001 ApJ, 554, 981

Pian, E., Mazzali, P. A., Masetti, N., et al. 2006 Nat, 442, 1010

Priddey, R. S., Tanvir, N. R., Levan, A. J., et al. 2006 MNRAS, 369, 1189

Prochaska, J. X., Gawiser, E., Wolfe, A. M., et al. 2003 ApJ, 595, L9

Prochaska, J. X., Bloom, J. S., Chen, H.-W., et al. 2004 ApJ, 611, 200

Prochaska, J. X., Chen, H.-W., Dessauges-Zavadsky, M., \& Bloom, J. S. 2007 ApJ, 666, 267

Prochter, G. E., Prochaska, J. X., Chen, H.-W., et al. 2006 ApJ, 698, L93

Rol, E., Wijers, R. A. M. J., Kouveliotou, C., et al. 2005 ApJ, 624, 868

Rol, E., van der Horst, A., Wiersema, K., et al. 2007 ApJ, 669, 1098

Ruiz-Velasco, A. E., Swan, H., Troja, E., et al. 2007 ApJ, 669, 1

Savaglio, S. 2006 New Journal of Phys., 8, 195 
Soderberg, A. M., Berger, E., Page, K. L. 2008, in press (arXiv:0802.1712)

Sollerman, J., Holland, S. T., Challis, P., et al. 2002 A\&A, 386, 944

Sollerman, J., Östlin, G. Fynbo, J. P. U., et al. 2005 NewA, 11, 103

Sollerman, J., Jaunsen, A. O., Fynbo, J. P. U., et al. 2006 AESA, 454, 503

Sollerman, J., Fynbo, J. P. U., Gorosabel, J., et al. 2007 A $\& A, 466,839$

Stanek, K. Z., Matheson, T., Garnavich, P. M., et al. 2003 ApJ, 591, L17

Stanek, K. Z., Gnedin, O. Y., Beacom, J. F., et al. 2006 AcA, 56, 333

Tanvir, N., Barnard, V. E., Blain, A. W., et al. 2004 MNRAS, 352, 1073

Thöne, C. C., Fynbo, J. P. U., Östlin, G., et al. 2008 ApJ, 676, 1151

van Paradijs, J., Groot, P., Galama, T. J., et al. 1997 Nat, 386, 686

van Paradijs, J., Kouveliotou, C., \& Wijers, R. A. M. J. 2000 ARA\&A, 38, 379

Vreeswijk, P. M., Ellison, S. L., Ledoux, C., et al. 2004 A\&A, 419, 927

Wiersema, K., Savaglio, S., Vreeswijk, P. M., et al. 2007 A $\& A, 464,529$

Wijers, R. A. M. J., Bloom, J. S., Bagla, J. S., \& Natarajan, P. 1998 MNRAS, 294, L13

Wolf, C. \& Podsiadlowski, P. 2007 MNRAS, 375, 1049

Woosley, S. \& Bloom, J. S. 2006 ARA\&A, 44, 507

\section{Discussion}

BURBIDGE: Some closeby -low redshift- GRBs are clearly associated with supernovaewe see traces of the SN light curves. But for most of the GRBs either no optical or radio object has been identified or only very faint afterglows are found. Thus the connection with galaxies- even star forming galaxies is much weaker. On the other hand, many optical spectra of GRB afterglows show the absorption features of QSOs. Thus I think that some attention should be paid to the connection of GRBs with QSOs. Here the MgII absorption seen in all of the afterglows with absorption but only in a fraction of QSOs presents a real problem for those who believe that the QSOs all have cosmological redshifts.

FYnBO: I would describe the situation as follows. For $z<2$ GRBs we almost always detect a host galaxy at the position of the afterglows. For more distant GRBs the fraction of hosts detected to a detection limit of about $R=27$ drops. The properties of the GRB absorption systems are most similar to the DLAs seen in QSO spectra although GRB systems on average are more metal rich. In no case has a GRB been associated directly with a galaxy hosting an AGN.

RAUw: There have been suggestions to use nuclear resonance absorption lines in the $\gamma$-ray spectrum to determine the redshifts of GRBs beyound $z=6$. That would require very large column densities, well in excess of the values you have presented. Can you comment on this?

FYNBO: I am afraid I do not have much insight into this issue. I can add that in some cases substantially higher column densities are inferred from X-ray absorption than from Hydrogen Lyman- $\alpha$ (e.g., Watson et al. 2007, ApJ, 660, L101). This is most likely due to ionized material close to the GRB progenitor. Still, the column densities inferred from X-ray absorption are so far all below $10^{23} \mathrm{~cm}^{-2}$ equivalent HI. 


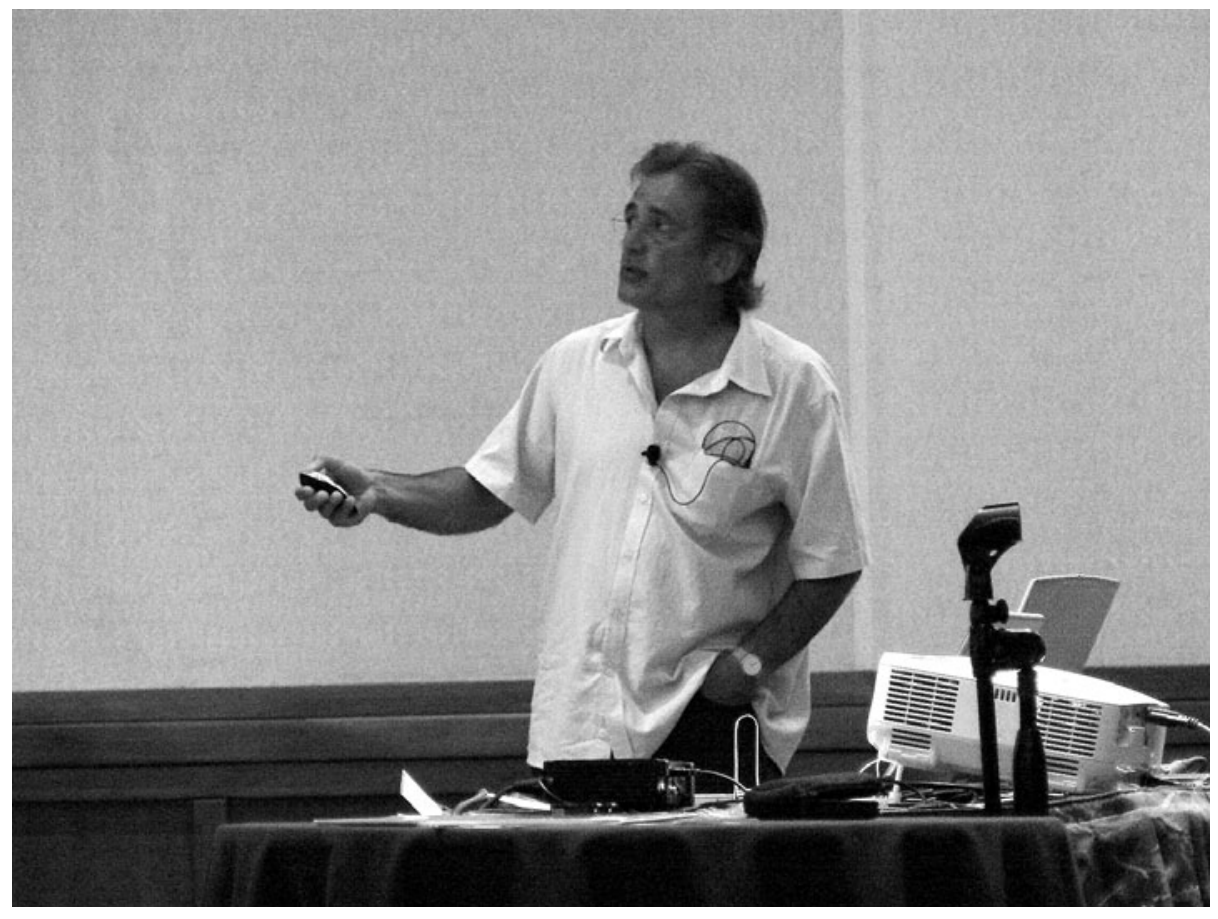

Max Pettini.

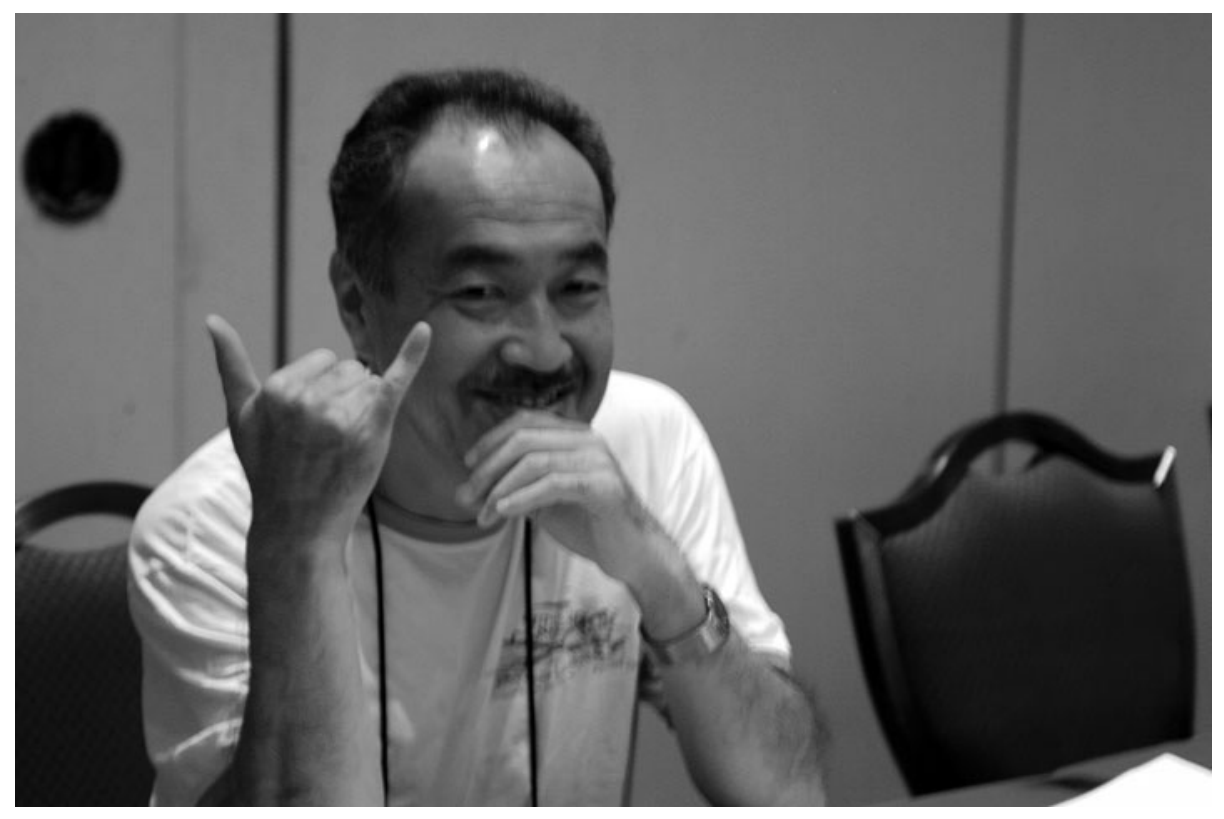

Yoshi Taniguchi. 